\title{
Dimensions of the language environment in infancy and symptoms of psychopathology in toddlerhood
}

Lucy S. King ${ }^{1}$, Francesca R. Querdasi ${ }^{1}$, Kathryn L. Humphreys ${ }^{2}$, \& Ian H. Gotlib ${ }^{1}$

${ }^{1}$ Stanford University, Department of Psychology, Stanford, CA, 94305, USA;

${ }^{2}$ Vanderbilt University, Department of Psychology and Human Development, Nashville, TN, 37235, USA

\section{Conflict of Interest}

The authors have no conflicts of interest to report.

\section{Data Availability}

Data are available at https://github.com/lucysking/lena_18mo_symptoms

\section{Acknowledgements}

Funding was provided by the National Institutes of Health (IHG, R21 MH111978; R21 HD090493); the National Science Foundation (LSK, Graduate Student Research Fellowship); and the Jacobs Foundation (KLH, Early Career Research Fellowship 2017-1261-05). We thank the team of research coordinators who assisted in data collection and management. We also thank the participants for their contributions.

\section{Corresponding author:}

Lucy S. King

Department of Psychology, Stanford University

Jordan Hall, 450 Serra Mall, Building 420

Stanford, CA 94305, USA

lucyking@stanford.edu 


\section{Research Highlights}

- Naturalistic measures of infants' experiences of language input obtained through passive monitoring are a promising method for measuring the early environment.

- We used the Language ENvironment Analysis (LENA) system to characterize the quantity and consistency of adult language input in 5- to 9-month-old infants' daily environments.

- Infants who experienced more consistent language input had lower symptoms of psychopathology approximately one year later, independent of other infant, maternal, and family characteristics.

- We discuss the implications of these findings for the measurement of infants' environments in relation to risk for psychopathology. 


\begin{abstract}
The quality of the early environment influences the development of psychopathology. Children who are deprived of sufficient environmental enrichment in infancy may be at higher risk for developing symptoms of psychopathology in toddlerhood. In this study, we investigated the prospective association between naturalistic measures of adult language input obtained through passive monitoring of infants' daily environments and emerging psychopathology in toddlerhood. In a sample of 100 mothers and their infants recruited from the community (mean age [range] $=6.73$ [5-9] months), we used the Language ENvironment Analysis (LENA) system to measure multiple dimensions of infants' language environments, including both the quantity and consistency of adult speech and conversational turns in infants' daily lives as well as the quantity of infant vocalizations. Subsequently, during toddlerhood (mean age [range] = 18.29 [17-21] months), mothers reported on their children's symptoms of psychopathology. Infants who experienced more consistent adult speech and conversational turns had lower symptoms of psychopathology in toddlerhood, independent of negative emotionality in infancy, maternal depressive symptoms, and laboratory-based measures of maternal sensitivity. These findings have implications for the measurement of environmental factors that may confer risk and resilience to emerging psychopathology.
\end{abstract}

Keywords: infancy, toddlerhood, psychopathology, caregiving, naturalistic, language 


\section{Introduction}

Over $20 \%$ of children ages 1-7 years suffer from psychiatric disorders (Vasileva, Graf, Reinelt, Petermann, \& Petermann, 2020). Unfortunately, psychopathology in young children is not transient; instead, approximately half of children with elevated symptoms in early life have persistent problems across development (Basten et al., 2016; Briggs-Gowan, Carter, BossonHeenan, Guyer, \& Horwitz, 2006). The quality of the early environment, which partially depends on the behavior of caregivers, influences the development of psychopathology (McGoron et al., 2012; van Aar, Leijten, Orobio de Castro, \& Overbeek, 2017; Yap et al., 2016). In particular, infants and young children who are deprived of sufficient environmental enrichment are at heightened risk for clinically significant psychopathology (Humphreys \& Zeanah, 2015), which can be observed in toddlerhood in the form of elevated internalizing symptoms and externalizing behaviors (Zeanah \& Zeanah, 2018; Zero to Three, 2016). Given that infants and young children have limited capacity to tell us about their experiences, many studies of the effects of variation in the early environment on the development of psychopathology use laboratory-based observations of caregiver behaviors to operationalize the quality of the environment.

There are several advantages of using laboratory-based observations of caregivers' behaviors during brief caregiver-child interactions to assess the quality of the early environment. Whereas self-report measures of caregiver behavior are subject to bias due to variation in caregivers' awareness of their own behaviors and the overreporting of socially desirable behaviors (Bornstein et al., 2015; Richards, Gilkerson, Xu, \& Topping, 2017), laboratory-based observations provide objective measures of caregivers' behaviors. Although identifying severe deviations in the early environment using child welfare records is also an objective method, laboratory-based observations measure variation in children's experiences along a continuum (Humphreys, King, \& Gotlib, 2018). Laboratory-based observations permit nuanced coding of the quality of caregivers' behaviors toward their children based on multiple modalities of communication. Finally, researchers can structure laboratory-based interactions to 
elicit specific types of caregiver behavior (e.g., sensitivity to child distress versus sensitivity during play; Leerkes, 2011), thereby enabling researchers to identify dimensions of the early environment that best predict child outcomes, including children's risk for the development of psychopathology (Davidov \& Grusec, 2006; Leerkes, Blankson, \& O’Brien, 2009).

It is important to note, however, that using laboratory-based assessments of caregiver behavior to operationalize children's experiences may not be ecologically valid (Belsky, 1980; Tamis-LeMonda, Kuchirko, Luo, Escobar, \& Bornstein, 2017). Indeed, the laboratory environment is a novel, often strange, setting for most caregivers and their children (Bronfenbrenner, 1977). The lack of competing demands that typify the environment outside the laboratory may result in "in-lab" caregiver behaviors that do not reflect infants' typical real-world experiences. Indeed, caregivers have been found to provide more attentive, responsive, and stimulating input in the laboratory than they do at home (Belsky, 1980), and to talk more to their infants and use more complex language during structured interactions than they do during their naturalistic routines (Tamis-LeMonda et al., 2017).

Concerns regarding the ecological validity of the laboratory context may be addressed in part by conducting semi-structured interactions in the home environment (e.g., video-recorded free-play interactions in families' living rooms), or by using checklists to rate caregivers' behaviors during 60- to 90-minute home interviews in which no formal caregiver-child interactions take place (e.g., the Infant-Toddler Home Observation for Measurement of the Environment [HOME] Inventory [Bradley \& Caldwell, 1984]); however, merely moving assessments into the home does not fully resolve concerns regarding the ecological validity of the behaviors observed. Such assessments may be susceptible to demand characteristics and social desirability effects due to the conspicuous presence of video cameras and/or researchers. Further, although children's experiences take place in many settings (e.g., while running errands, on the playground, during mealtimes) and across many hours in a day, both laboratory-based assessments and semi-structured or interview-based home assessments are 
location- and time-limited. Historically, the high cost of recording and coding behavior in daily life has restricted researchers' ability to conduct naturalistic assessments of the early environment (Mehl, 2017). Recently, however, technological advances have facilitated passive monitoring of infants' auditory environments while families move through their daily lives. Given that intensive auditory monitoring cannot be coded in the same manner as brief laboratory-based observations and that infants' experiences are also influenced by non-audible caregiver behavior (e.g., facial expression), measures of infants' auditory environments do not mimic laboratory-based measures of caregiver behavior, nor do they fully capture infants' experiences. Nonetheless, passive monitoring of auditory stimulation does capture aspects of infants' experiences, making this a promising alternative or complementary method for operationalizing the nature of infants' early environments.

The Language ENvironment Analysis (LENA) wearable digital device and software enables day-long recordings of infants' naturalistic speech environments and fast and reliable quantification of ambient adult speech and adult-infant vocal exchanges (i.e., "conversational turns") in infants' daily lives (Gilkerson \& Richards, 2008). The LENA system has several potential advantages for measuring the behaviors of adults in children's environments. First, monitoring of adult language input using the LENA is passive and unobtrusive, thereby likely reducing biases due to the effects of observation. Second, compared to time-intensive observational coding of laboratory-based interactions (e.g., coding of a ten-minute interaction may require an hour of an expert coder's time), the LENA allows for efficient, albeit less nuanced, scoring of large quantities of data (Cychosz et al., 2020). Third, in contrast to laboratory-based observations that typically focus on the behavior of a single caregiver (usually the mother), the LENA records language input from multiple caregivers (e.g., mothers, fathers, other family, and childcare professionals) delivered across locations for up to 16 hours, arguably yielding more ecologically-valid measures of infants' environments that reflect the broader availability and engagement of caregivers in infants' daily lives. Finally, data collected via LENA 
recordings can be used to generate multiple measures of infants' environments that may explain unique variance in children's outcomes. For example, previous studies using the LENA to examine children's cognitive functioning have distinguished between measures of overheard adult speech and conversational turns (Ganek \& Eriks-Brophy, 2018), often reporting that the quantity of conversational turns is associated more strongly with cognitive functioning than is the quantity of overheard adult speech (Gilkerson et al., 2018; Merz, Maskus, Melvin, He, \& Noble, 2019). Although researchers have focused on the quantity of adult words and conversational turns regardless of their distribution across the day, data obtained via LENA recordings can also be used to measure the consistency of adult words and conversational turns, or the regularity with which the target child experiences any overheard language input or vocal engagement, respectively. The consistency of language input from adults is a marker of the extent to which caregivers are physically available to initiate or respond to infants' verbal and nonverbal signals (adult word consistency) or of the extent to which caregivers vocally engage with their infants on a regular basis throughout the day (conversational turn consistency).

Measures of the consistency of language input may be particularly useful when investigating risk for symptoms of psychopathology. Receiving input from caregivers on a consistent basis is theorized to be a species-expectant experience necessary for the development of both socioemotional and cognitive skills (Bowlby, 1988; McLaughlin, Sheridan, \& Nelson, 2017). From the perspective of infants, cumulative experiences of the availability of adults may influence how their representations of their environments develop (Johnson, Dweck, \& Chen, 2016; Sherman, Rice, \& Cassidy, 2015). For example, an infant who experiences language input on a consistent basis may form different expectations about whether an adult is available to notice and respond to their cues than would an infant who does not experience consistent input. In turn, infants' experience-based representations of their environments likely affect their emotions (i.e., the processes that help them evaluate and respond to their worlds; Cole, Martin, \& Dennis, 2018) thereby shaping their behavior and influencing the emergence of 
psychopathology (Sherman et al., 2015). In fact, there is extensive evidence that infants who experience deprivation of consistent input from caregivers in the form of institutional care are at risk for the development of psychopathology (Gunnar, Van Dulmena, \& The International Adoption Project Team, 2007; Hawk \& McCall, 2010; Humphreys et al., 2015). In studies of institutionalized infants, however, the deprivation of input is usually inferred based on scientists' knowledge of the nature of institutional care. Considerably less research has examined the consistency of adult input in typical family-based settings due to challenges in directly measuring this construct along a continuum in infants' daily environments. Despite the potential advantages of the LENA system for measuring infants' environments, including the extent to which adults provide consistent language input, researchers have not yet used this technology to examine individual differences in risk for the development of psychopathology. The goal of the current study was to examine whether measures of infants' experiences of adult language input in their daily lives are meaningfully related to the development of symptoms of psychopathology, and, if so, to determine which dimensions of language input are most strongly associated with symptoms of psychopathology.

In this study, we examined the longitudinal associations between naturalistic measures of infants' environments operationalized through passive monitoring of daily language input at age 6 months and parent-reported symptoms of psychopathology at age 18 months. Specifically, we used the LENA system to measure multiple dimensions of infants' language environments, including the quantity and consistency of adult speech and conversational turns in infants' daily lives as well as the quantity of infant vocalizations. Given precedent for using laboratory-based observations of caregiver behavior to measure infants' environments, we also obtained measures of maternal sensitivity during infant distress and during play in the laboratory environment.

We examined the prospective relations between the naturalistic measures of language input and emerging psychopathology, independent of infant temperament, maternal depressive 
symptoms, and family socioeconomic status. To account for the possibility that infants who vocalize more are better able to elicit input from their caregivers and/or achieve earlier social and cognitive competencies that lead to a positive developmental cascade (Masten \& Cicchetti, 2010), we also examined the quantity of infant vocalizations. Finally, to determine whether the naturalistic measures of infants' language environments obtained using the LENA system predicted unique variance above and beyond prevailing measures of the early environment, we examined the laboratory-based measures of maternal sensitivity in infancy as covariates. We hypothesized that the consistency of adult words and conversational turns are more strongly negatively associated with symptoms of psychopathology in toddlerhood than are the quantity of adult speech and conversational turns.

\section{Method}

\section{Participants}

Participants were women and their children who were recruited from communities in the San Francisco Bay Area to participate in the Brain and Behavior Infant Experiences (BABIES) project (Humphreys, King, Choi, \& Gotlib, 2018), an observational study of the association between perinatal experiences and infant and toddler psychobiological development. The sample for the current analyses included mother-child dyads who completed assessments of the child's caregiving environment at age 5-9 months (infant assessment) and an online followup assessment of the child's symptoms of psychopathology at age 17-21 months (toddler assessment). Of the 155 dyads who provided any data at the infant assessment, 8 did not complete the toddler assessment by the time of the current analysis, and an additional 47 either did not participate in the LENA assessment or did not provide usable LENA recordings for the naturalistic assessment of infants' environments (42 dyads did not complete, 3 dyads completed when the infant was $>9$ months old, and 2 dyads completed incorrectly), yielding a final sample of 100 dyads. Descriptive statistics for the final sample are presented in Table 1.

\section{Procedure}


The BABIES Project was approved by the Stanford Institutional Review Board. Mothers provided informed written consent for themselves and their infants and were compensated for their time. Participants included in the current analyses were recruited either during their pregnancies (16-35 weeks gestation) or when their infants were age 6 months or younger through online advertisements and flyers posted in the local community. Participants recruited during pregnancy participated in additional sessions not included in the current analyses. All participants were screened for inclusion/exclusion criteria through a phone interview. When infants were approaching age 6 months, mother-infant dyads were invited to attend a laboratory session to conduct the infant assessment. Inclusion criteria for this session were that mothers had a singleton infant between ages $\geq 5$ and $<9$ months, were $\geq$ age 18 years, were fluent in English, and had no immediate plans to leave the geographic area. Exclusion criteria included maternal bipolar disorder, maternal psychosis, maternal severe learning disabilities, severe complications during birth, infant head trauma, infant premature birth (prior to 36 weeks gestation), infant congenital/genetic/neurological disorders, and contraindication for infant magnetic resonance imaging. At the infant assessment, dyads participated in two laboratorybased video-recorded mother-infant interactions and mothers completed questionnaires and interviews. At the end of this session, a research coordinator provided dyads with a wearable (for the infant) audio recording device to assess the infants' daily language environment (described below). When children were approaching age 18 months, mothers were contacted to complete a series of online questionnaires about their child (toddler assessment). Questionnaire data were collected and managed using REDCap electronic data capture tools hosted at Stanford University (Harris et al., 2009, 2019).

\section{Measures}

Measures in the public domain are available at https://osf.io/2gp6b/?view only $=$ 4381f7d2a4cf40b1abcf0b41f76cdf63. We provide additional details concerning the measures in the Supplementary Material. 


\section{Infant Assessment}

Naturalistic Language Environment. As previously described (King, Camacho, Montez, Humphreys, \& Gotlib, 2020), we provided mother-infant dyads with a Language ENvironment Analysis (LENA) digital processing device and specialized infant clothing with written and oral instructions that the infant wear the device from waking to bedtime on a typical day at home. The LENA device records for up to 16 hours. To be included in the current analyses, we required families to have completed $\geq 8$ hours of recording. After completing recording, we emailed mothers a link to complete a set of basic follow-up questions about their recording experience, who was with the baby on the day of the recording, and the percent of the day $(0-100 \%)$ that the mother spent with the infant.

We uploaded the recordings to the LENA analysis software (Xu, Yapanel, \& Gray, 2009), which yields estimates of the number of adult words (AWs; i.e., the number of overheard "near and clear" words spoken by adults in the infant's vicinity), conversational turns (CTs; the number of adult-infant or infant-adult back-and-forth vocalizations), and infant vocalizations at the level of five-minute epochs. Using these estimates, we computed the following measures:

1) To measure the quantity of AWs and CTs we computed the maximum number of AWs and maximum number of CTs within a 1-hour period. This approach is consistent with previous studies use the LENA system (Brito et al., 2020; Romeo, Leonard, et al., 2018; Romeo, Segaran, et al., 2018) and minimized overlap between measures of quantity and consistency in the current study.

2) To measure the consistency of exposure to AWs and CTs, we computed the proportion of five-minute epochs out of the total number of five-minute epochs in the recording in which $\geq 1 \mathrm{AW}$ (AW consistency) or $\geq 1 \mathrm{CT}$ (CT consistency) was present. 
3) Finally, to measure the infant's capacity to vocalize, we computed the maximum number of infant vocalizations within a 1-hour period.

Laboratory Maternal Sensitivity. Mothers and infants participated in two laboratorybased caregiving interactions. We used separate cameras focused on the mother and the infant to record the interactions for subsequent observational coding.

First, as previously described (Humphreys, King, Choi, et al., 2018; King, Humphreys, \& Gotlib, 2019), dyads completed the ten-minute laboratory-based repeated Still-Face Paradigm (SFP-R; Haley \& Stansbury, 2003; Tronick, Als, Adamson, Wise, \& Brazelton, 1978). The SFP$R$ is a caregiver-child interaction stressor that reliably elicits infant distress (Mesman, van IJzendoorn, \& Bakermans-Kranenburg, 2009), an effect we replicated in the current sample (Camacho et al., 2019). Specifically, the SFP-R consists of five two-minute face-to-face interaction episodes between mother and child: (1) a baseline normal play episode; (2) the stillface episode in which mothers become unresponsive and maintain a neutral expression without touching their child; (3) a reunion episode in which mothers resume normal interaction; (4) a second still-face episode; and (5) a final reunion episode. Following the SFP-R, dyads completed an eight-minute "free play" interaction. Dyads were seated on a playmat on the floor with access to a box of age-appropriate toys and were instructed to play as they usually would at home.

Using the infant adaptation of the Parent-Child Interaction Rating Scales (Bosquet Enlow et al., 2014; Sosinsky et al., 2004), trained independent coders rated maternal sensitivity during each 30-second interval of the SFP-R and each 2-minute interval of the free play; possible scores ranged from 1 (not at all characteristic) to 7 (very characteristic), increasing in half-point increments. Sensitivity was defined as the extent to which the mother demonstrated awareness of her infant's cues (e.g., needs, moods, interests) and responded to these cues contingently and appropriately (Sosinsky et al., 2004). Using coder ratings during the SFP-R and the free play, we calculated the following measures: 
1) To quantify maternal sensitivity during infant distress, we computed the mean rating of sensitivity across all 30-second intervals in the two reunion episodes of the SFP-R (Conradt \& Ablow, 2010; Grant, McMahon, Reilly, \& Austin, 2010).

2) To quantify maternal sensitivity during play, we computed the mean rating of sensitivity across all 2-minute intervals of the free play (Leerkes, 2011).

To assess reliability, randomly selected subsets of the SFP-R $(N=17)$ and free play $(N=24)$ videos were rated by two coders. Reliability was good at the level of mean ratings for both sensitivity to distress (ICC $=.87)$ and sensitivity during play $(I C C=.85)$. Finally, to confirm that the free play did not elicit infant distress, coders rated infant negative mood using the PCIRS-IA on a scale of 1 (very low) to 7 (very high); on average, negative mood was between very low and low (mean[SD] = 1.61 [0.82]). Six dyads did not provide usable data for the assessment of maternal sensitivity during infant distress and four dyads did not provide usable data for the assessment of maternal sensitivity during play (see Supplementary Material).

Infant negative emotionality. Mothers completed the Short Form version of the Infant Behavior Questionnaire-Revised (IBQ-R-SF; Putnam, Helbig, Gartstein, Rothbart, \& Leerkes, 2014). The IBQ-R-SF, which includes 91 items and several subscales, asks parents to rate on a scale from 1 (never) to 7 (always) the extent to which their infant exhibited various behaviors over the past seven days. The negative affectivity scale of the IBQ-R-SF is composed of the sadness, distress to limitations, fearfulness, and falling reactivity subscales (Cronbach's $\alpha=$ .88). We focused on this dimension of temperament given that negative affectivity is a broadband risk factor for the development of symptoms of psychopathology (KostyrkaAllchorne, Wass, \& Sonuga-Barke, 2020). Four mothers did not complete the IBQ-R-SF.

Maternal Depressive Symptoms. Mothers completed the Center for Epidemiological Studies Depression Scale (CES-D; Radloff, 1977). The CES-D, which includes 20 items, instructed mothers to consider their past week and respond to items on a 4-point scale from 0 (rarely or none of the time) to 3 (most or all of the time), with higher scores representing greater 
symptoms of depression $(\alpha=.92)$. At the six-month assessment, 16 percent of mothers had CES-D scores $\geq 16$, indicating risk for clinical depression.

Socioeconomic Status. We used measures of maternal education and family income to operationalize socioeconomic status. Mothers reported their education level using an ordinal scale ranging from 0 (No schooling completed) to 9 (Graduate degree). Mothers reported annual household income in bins ranging from $1(\$ 0-50,000)$ to $7(>\$ 150,000)$, and the number of people in their household. We calculated family income-to-needs ratio by dividing the annual household income (median point of each bin) by the U.S. Department of Housing and Urban Development's (HUD; www.huduser.gov) 2017 county-specific low-income threshold for the number of people in the household. For comparability with studies of poverty in other geographic regions, we also calculated the income-to-needs ratio based on the federal poverty thresholds (www.census.gov). The HUD low-income limits and the federal poverty thresholds are posted at: https://osf.io/2gp6b/?view only=4381f7d2a4cf40b1abcf0b41f76cdf63.

\section{Toddler Assessment}

Toddler Symptoms of Psychopathology. Mothers completed an abbreviated version of the Infant-Toddler Social-Emotional Assessment (ITSEA; Carter, Briggs-Gowan, Jones, \& Little, 2003). The ITSEA, which includes several subscales covering four domains (internalizing, externalizing, dysregulation, and competence), asks parents to rate the extent to which their child exhibits various behaviors on a scale of 0 (not true/rarely) to 2 (very true/often). Each subscale is scored by taking the mean of responses. In the current study, we administered eight of the ITSEA subscales, including at least one subscale from each of the four domains. We computed a total score for symptoms of psychopathology by summing the mean scores for the following scales: internalizing (depression/withdrawal, general anxiety), externalizing (activity/impulsivity, aggression/defiance), and negative emotionality ( $\alpha=.83)$. A factor analysis indicated that this approach was appropriate for our data (presented in the Supplementary 
Material). Twenty percent of toddlers had symptoms of depression/withdrawal, general anxiety, activity/impulsivity, aggression/defiance, or negative emotionality above age and gendernormed cutoffs for clinical concern.

Maternal Depressive Symptoms. Mothers again completed the CES-D to assess depressive symptoms in the past week $(\alpha=.90)$. At the toddler assessment, 14 percent of mothers had CES-D scores $\geq 16$, indicating risk for clinically significant depression.

\section{Data Analysis}

Scoring scripts are available at https://github.com/babies-study/babies scoring and primary analysis scripts and analyzed data are available at https://github.com/lucysking/lena $18 \mathrm{mo}$ symptoms. All analyses were conducted in R version 4.0.2 (R Core Team, 2020).

\section{Associations of the Dimensions of the Language Environment in Infancy with Symptoms of Psychopathology in Toddlerhood}

First, we conducted separate ordinary least squares (OLS) linear regression models testing the association between each measure of the language environment (AW quantity, CT quantity, AW consistency, CT consistency, infant vocalizations) and symptoms of psychopathology in toddlerhood. We tested each measure separately given that these measures were moderately to highly positively intercorrelated and would violate the assumption of non-collinearity if entered together in an OLS regression model. To identify which measures of language input were most strongly associated with symptoms of psychopathology in toddlerhood, we compared standardized estimates and Bayes Factors (Morey, 2019) for each association. Bayes Factors quantify the strength of evidence in support of the alternative hypothesis (i.e., that the measure of language input is associated with symptoms of psychopathology) versus the null hypothesis (i.e., that the measure of language input does not explain variance beyond a covariate-only model). 
Prior to conducting each of the OLS linear regression models, we identified covariates associated with symptoms of psychopathology in toddlerhood using formal model fitting procedures (Chambers, 1992). Specifically, we started with an intercept-only model and then iteratively added covariates, comparing the fit of models with and without each covariate using the anova() function in $\mathrm{R}$. We retained covariates that significantly improved model fit in the final best fitting model. We tested the following 13 covariates: infant negative emotionality, infant age, infant sex, maternal depressive symptoms at the infant and toddlerhood assessments, maternal age, maternal race (White vs. person of color), maternal Hispanic or Latinx ethnicity, maternal education, family income-to-needs ratio based on the HUD low-income limits, family income-to-needs ratio based on the federal poverty thresholds, and the duration of the analyzed LENA recording.

Second, to complement the results of the separate OLS linear regression models, we conducted an elastic net regression—a form of regularized regression—using the "glmnet" package (Jerome, Hastie, Simon, \& Tibshirani, 2010) in which we entered all four measures of language input (AW quantity, CT quantity, AW consistency, CT consistency), the quantity of infant vocalizations, and the identified covariates as predictors of symptoms of psychopathology in toddlerhood. Importantly, the elastic net regression model is robust to collinearity, retaining correlated predictors provided that they minimize error (Zou \& Hastie, 2005). Model complexity is penalized using $\lambda$, the regularization parameter, which shrinks the coefficients of correlated predictors towards each other. We employed a full EN penalty $(\alpha=0.50)$ and an expected gaussian distribution. We used leave-one-out cross-validation to identify the $\lambda$ value corresponding to the optimal model (i.e., the $\lambda$ that minimized mean squared error), which yields a sparse matrix of non-zero coefficients. To aid interpretation of the elastic net coefficient estimates, we computed zero-order effect sizes (Pearson's $r$-values) for the associations between the variables that were selected by the elastic net model and symptoms of psychopathology in toddlerhood. 


\section{Additional Analyses}

Next, given that laboratory-based observations are the prevailing method for objectively measuring the caregiving environment, we conducted OLS linear regression models to determine whether the naturalistic measures of language input that we identified as most strongly associated with symptoms of psychopathology in toddlerhood predicted unique variance above and beyond the laboratory-based measures of maternal sensitivity during infant distress and maternal sensitivity during play. Finally, given that previous studies have found that indices of family socioeconomic status reflecting greater affluence are positively associated with measures of language input obtained using the LENA system (Fernald, Marchman, \& Weisleder, 2013; Merz et al., 2019), we also tested associations among maternal education (Spearman's $r h o$ ), family income-to-needs ratio (Pearson's $r$ ), and measures of the quantity and consistency of language input.

\section{Results}

We present descriptive statistics for the study sample in Table 1 and histograms for each of the naturalistic measures of language input, laboratory-based measures of maternal sensitivity, and toddler symptoms of psychopathology in the Supplementary Material. Mothers were racially and ethnically diverse and tended to be highly educated. Based on the HUD lowincome limits, which take into account median income and housing costs in the geographic region in which data were collected, $22 \%$ of the sample was "low income." Based on the federal poverty thresholds, $3 \%$ of the sample was in poverty and $12 \%$ was "low income" (i.e., $\leq 200 \%$ of the federal poverty threshold). In Figure 1, we present Pearson's correlation coefficients for associations among the naturalistic measures of language input and the laboratory-based measures of maternal sensitivity. None of the naturalistic measures of language input was significantly associated with either of the laboratory-based measures of maternal sensitivity, nor were the laboratory-based measures of maternal sensitivity during infant distress and during play significantly correlated with each other. However, the measures of language input were 
positively intercorrelated. Although AW consistency was not significantly associated with AW quantity, CT quantity, or infant vocalizations, it was strongly positively associated with CT consistency. Further, both CT consistency and CT quantity were positively associated with each other and with infant vocalizations. To assist readers in differentiating the consistency of language input from the quantity of language input, Figure 2 presents the distributions of CTs across the day for two infants: one exposed to lower quantity but higher consistency, and one exposed to lower consistency but higher quantity. In the Supplementary Material, we present correlations between each measure of language input and variability in the LENA recording protocol (i.e., duration of recording, start and end times of recording)

\section{Associations of the Dimensions of the Language Environment in Infancy with Symptoms of Psychopathology in Toddlerhood}

The results of formal model fitting procedures testing each potential covariate of symptoms of psychopathology in toddlerhood indicated that only the inclusion of infant negative emotionality and maternal symptoms of depression at the toddler assessment significantly improved model fit (see the Supplementary Material for additional information). Therefore, infant negative emotionality and maternal symptoms of depression at the toddler assessment were included as covariates in all subsequent models. Infant negative emotionality was positively associated with symptoms of psychopathology in toddlerhood $(\beta=0.33,95 \% \mathrm{Cl}[0.14,0.52]$, SE $\left.=0.10, t(93)=3.42, p<.001, R^{2}=.11\right)$ as were maternal symptoms of depression at the toddler assessment $\left(\beta=0.18,95 \% \mathrm{Cl}[-0.01,0.37], \mathrm{SE}=0.10, t(93)=1.84, p=.069, R^{2}=.03\right)$.

We present the results of each of the four OLS linear regression models testing each of the measures of language input and infant vocalizations in Table 2. The consistency of adult words was negatively associated with symptoms of psychopathology when covarying for infant negative emotionality as was the consistency of conversational turns (see Figure 3). Both of these associations had small standardized effect sizes $\left(\beta_{\text {AW }}\right.$ consistency $=-0.22, \beta_{\mathrm{CT}}$ consistency $=-$ 0.25 ) and Bayes Factors indicating "moderate" or "positive" evidence for the alternative 
hypothesis that they explained meaningful variation in symptoms of psychopathology above and beyond the covariates (Kass \& Raftery, 1995; Quintana \& Williams, 2018). However, the evidence in favor of CT consistency as a predictor of symptoms of psychopathology was stronger than for $\mathrm{AW}$ consistency $\left(\mathrm{BF}_{\mathrm{AW} \text { Consistency }}=3.00, \mathrm{BF}_{\mathrm{CT}}\right.$ consistency $\left.=5.79\right)$. Although the quantity of adult words was not associated with symptoms of psychopathology in toddlerhood, CT quantity was negatively associated with symptoms $\left(\beta_{\text {Ст Quantity }}=-0.22\right)$. The Bayes Factor for CT quantity, however, was $<3$ indicating weak evidence for the alternative hypothesis that this dimension of language input was associated with symptoms of psychopathology. Finally, the quantity of infant vocalizations was negatively associated with symptoms of psychopathology with moderate evidence in favor of this alternative hypothesis $\left(\beta_{\text {Infant }}\right.$ vocalizations $=-0.24, \mathrm{BF}_{\text {Infant }}$ Vocalizations $=4.73)$

Results of the elastic net regression in which we entered AW consistency, CT consistency, AW quantity, CT quantity, infant negative emotionality, maternal depressive symptoms at the toddler assessment, and the quantity of infant vocalizations together as predictors of symptoms of psychopathology in toddlerhood indicated that each of these variables, except for AW quantity, helped to minimize error in the prediction of symptoms of psychopathology (see Table 3). Thus, despite moderate to high correlations among measures of multiple dimensions of infants' language environments, these measures appear to contribute uniquely to the prediction of psychopathology in toddlerhood. Collectively, the variables selected by the elastic net regression explained $24 \%$ of the variance in symptoms of psychopathology in toddlerhood.

\section{Additional Analyses}

We examined whether the associations between AW consistency and CT consistencythe measures of language input most strongly associated with symptoms of psychopathology in toddlerhood-remained when accounting for variation in the laboratory-based measures of maternal sensitivity during infant distress and during play. In an OLS linear regression model 
including infant negative emotionality and maternal depressive symptoms at the toddler assessment, AW consistency remained similarly associated with symptoms of psychopathology in toddlerhood when covarying the laboratory-based measures of maternal sensitivity $(\beta=-0.22$, $\left.95 \% \mathrm{Cl}[-0.41,-0.03], t(83)=-2.26, p=.026, R^{2}=.05\right)$. Somewhat surprisingly, neither maternal sensitivity during infant distress nor maternal sensitivity during play was significantly associated with symptoms of psychopathology in toddlerhood when covarying for the other variables in the model (sensitivity to distress: $\beta=0.08,95 \% \mathrm{CI}[-0.11,0.27], t(83)=0.88, p=.382$; sensitivity during play: $\beta=-0.11,95 \% \mathrm{Cl}[-0.31,0.10], t(83)=-1.04, p=.300)$ or when examining the zero-order correlations (sensitivity to distress: $r(88)=.11,95 \% \mathrm{Cl}[-.10, .31], p=.303$; sensitivity during play: $r(94)=-.19,95 \% \mathrm{CI}[-.37, .01], p=.068)$. Results were similar with respect to CT consistency. In an OLS linear regression model including the three variables of CT consistency, infant negative emotionality, and maternal depressive symptoms (infant vocalizations were not included in this model due to collinearity with CT consistency), CT consistency remained similarly associated when covarying the laboratory-based measures of maternal sensitivity $\left(\beta=-0.23,95 \% \mathrm{CI}[-0.42,-0.04], t(83)=-2.43, p=.017, R^{2}=.06\right)$.

None of the measures of the quantity and consistency of language input was significantly associated with maternal education or income-to-needs ratio based on HUD lowincome limits (correlation values $<|.18|, p$-values $>$.066). Findings with respect to the association of the consistency of language input with symptoms of psychopathology in toddlerhood were highly similar when covarying for maternal education and family income-to-needs ratio. Both AW and CT consistency remained significantly negatively associated with symptoms of psychopathology in toddlerhood (AW consistency: $\beta=-0.21, \mathrm{SE}=0.10,95 \% \mathrm{Cl}[-0.40,-0.02]$, $t(90)=-2.15, p=.034, \Delta R^{2}=.04 ; \mathrm{CT}$ consistency: $\beta=-0.23, \mathrm{SE}=0.10,95 \% \mathrm{Cl}[-0.42,-0.03], t(91)=-$ 2.30, $\left.p=.025, \Delta R^{2}=.05\right)$.

\section{Source of Naturalistic Language Input}


A key difference between naturalistic measures of language input obtained through the LENA system and laboratory-based measures of language input is that whereas the naturalistic measures capture input from any adult, laboratory-based measures usually capture input from only one caregiver (usually the mother). Therefore, a possible explanation for the finding that naturalistic measures of language input were associated with symptoms of psychopathology in toddlerhood whereas our laboratory-based measures were not is that AW and CT consistency reflect the input from adults across a network of caregivers who collectively contribute to infants' experiences. To clarify the origin of speech in infants' environments, we examined responses to the follow-up questionnaire that mothers completed following the LENA recording. Among the infants whose mothers responded to all questions on the follow-up questionnaire ( $N=74,74 \%)$, all were reported to have spent time with their mother on the day of the recording and $88 \%$ spent time with both their mother and their father (or mother's significant other) on the day of recording. Beyond infants' parents, the most common adult present was a grandparent (28\%), followed by a family friend (18\%), or another relative $(8 \%)$. Only $8 \%$ of infants spent time at daycare and $5 \%$ spent time with a babysitter/nanny. Thus, although we captured input from multiple potential caregivers using the LENA device, it appeared that infants' parents were the dominant source of input. On average, mothers reported spending $82 \%$ of the recording day with their infant with a modal response of $100 \%(S D=24.04$, median=91\%, range $=10-100 \%)$. Neither the percentage of time that mothers spent with their infants on the recording day ( $\mathrm{rho}=.08, p=.479)$ nor the number of adults present during the recording day (rho=-.10, $p=.377)$ was associated with symptoms of psychopathology in toddlerhood.

\section{Discussion}

The goal of this study was to examine whether measures of infants' experiences with adult language input in their daily lives are meaningfully related to the development of symptoms of psychopathology in toddlerhood, and, if so, to determine which dimensions of language input are most strongly associated with subsequent symptoms of psychopathology. 
We used the LENA digital recording device and software to naturalistically measure multiple dimensions of infants' language environments, including both the quantity and consistency of adult speech and conversational turns (i.e., adult-infant vocal exchanges) in infants' daily lives as well as the quantity of infant vocalizations. Further, we collected laboratory-based measures of maternal sensitivity during infant distress and during play. The complementary results of separate linear regression analyses and an automatic variable selection technique that is robust to multicollinearity indicated that infants who experienced more consistent exposure to adult speech and conversational turns in daily life had lower symptoms of psychopathology in toddlerhood. Both the consistency of adult words and conversational turns predicted symptoms in toddlerhood above and beyond laboratory-based measures of maternal sensitivity, maternal reports of infant negative emotionality, maternal depressive symptoms in toddlerhood, maternal education, and family income-to-need ratio.

The associations between AW and CT consistency had Bayes Factors indicating "positive" evidence in favor of the hypothesis that the consistency of adult speech and conversational turns predicted symptoms of psychopathology. Overall, based on effect size, the consistency of conversational turns was the strongest predictor of symptoms of psychopathology in toddlerhood relative to other measures of infants' language environments. The standardized effect size for the association between CT consistency and symptoms of psychopathology in toddlerhood was -0.25 , explaining $6 \%$ of the variance. This effect size is in the small to medium range based on Cohen's standards for interpreting effect sizes, where $r$ values of .10, .30, and .50 correspond to small, medium, and large effects (Cohen, 1977, 1988). One way to understand the meaning of a "small" effect is to compare it to the magnitude of other associations that are already well understood (Funder \& Ozer, 2019). The magnitude of the association between CT consistency and subsequent symptoms of psychopathology is similar to or larger than those observed in meta-analyses of the effects of family socioeconomic status on psychopathology in childhood (Peverill et al., 2021; $r$-values ranging from .10-.16) and of the 
effects of early life adversity on risk for major depressive disorder in childhood (LeMoult et al., 2020; average $r$-values range from .05 to .29). In the current study, we did not identify statistically significant associations between laboratory-based observations of maternal sensitivity and symptoms of psychopathology in toddlerhood. Findings from large longitudinal cohorts have identified small associations of maternal sensitivity in infancy with subsequent symptoms of psychopathology (Kok et al., 2013; Wright, Hill, Sharp, \& Pickles, 2018; r-values ranging from -.30-.13). Therefore, our findings suggest that measures of language input in infancy are similarly important for predicting the development of psychopathology as are other established indices of the quality of the early environment. Beyond prevailing methods for operationalizing variation in infants' experiences (i.e., laboratory-based observations of caregiver behavior), the LENA technology may be leveraged to examine signals in infants' environments that appear to be important for their subsequent socioemotional functioning.

Although researchers have used the LENA system to measure the early language environment in order to examine vulnerability to lower levels of cognitive functioning (Gilkerson et al., 2018; Weisleder \& Fernald, 2013), the current study is the first to leverage this technology to investigate these tools in the longitudinal prediction of psychopathology. We posited that measures of the language environment obtained using the LENA may reflect not only the amount of cognitive stimulation an infant receives but-relevant to socioemotional development-the broader availability of caregivers. Recent research using a novel device to passively monitor the physical proximity of caregivers and their infants supports this formulation; infants who experience more adult words and conversational turns spend a greater proportion of their day within touching distance of their caregivers (Salo et al., 2020). We proposed that the consistency of adult speech reflects the extent to which adults are physically available to initiate or respond to infants' verbal and nonverbal signals (AW consistency) or the extent to which adults vocally engage with their infants on a regular basis throughout the day (CT consistency). Receiving consistent input from caregivers is posited to be fundamental to the development of 
socioemotional skills (Bowlby, 1988; McLaughlin et al., 2017). When caregivers are consistently available, infants learn that they will receive responses to their verbal and nonverbal signals, and, gradually, they integrate these experiences into the development of effective emotion regulation skills (Calkins, 2011; Calkins \& Hill, 2007). Perhaps the strongest evidence for the importance of available and engaged caregivers comes from studies of institutionalized infants, who likely experience low amounts of, and inconsistent, input from adults (Zeanah, Smyke, \& Settles, 2006). Several studies indicate that these children are at heightened risk for poorer emotion regulation and, ultimately, the development of psychopathology (Gunnar et al., 2007; Hawk \& McCall, 2010; Humphreys et al., 2015).

We and others have previously discussed the challenges of measuring neglect during infancy among children raised in their families of origin (Humphreys, King, \& Gotlib, 2018; Stoltenborgh, Bakermans-Kranenburg, \& Van ljzendoorn, 2013), positing that psychosocial neglect is best represented as variations along a continuum from severely neglectful to highly enriched environments (King et al., 2019). Although we cannot draw strong conclusions based on a single day of observation, our results highlight the importance of building a program of research that investigates variation in infants' experiences of naturalistic language input in relation to risk for psychopathology. Initially, this research program should focus on developing a more nuanced understanding of what aspects of infants' environments are captured by these naturalistic measures. Although our results supported our hypothesis that the consistency of language input is more strongly associated with symptoms of psychopathology than is the quantity of this input, the design of this study does not allow us to identify the mechanisms for this association. Based on previous theory and research, we posit that infants who experience language input on a consistent basis are more likely to develop representations of their environments in which adults are available to notice and respond to their cues (Johnson et al., 2016; Sherman et al., 2015). In turn, these representations may affect their emotions and behaviors (Sherman et al., 2015). Other developmental processes (e.g., maturation of executive 
function), however, could mediate the association between the consistency of language input and symptoms of psychopathology.

As previously discussed, although using the LENA system to operationalize infants' environments enhances ecologically validity, measures obtained from the LENA system are less nuanced measures of caregiver behavior than are those derived from coding of laboratorybased interactions. Information about facial expression, content of speech, and tone of voice paired with LENA recordings is likely to yield a more precise understanding of infants' daily experiences with adults. In addition to reflecting the broader availability of adults, AW consistency captures adult words that are not directed to signals from the infant, including conversations among adults in the infant's proximity who may not be aware of what the infant is doing. An open question is whether any language input indicating the availability of adults contributes to the developmental processes that reduce risk for psychopathology, or whether only language input that is contingent with infants' signals is operative. Although CT consistency directly captures caregivers' engagement with their infants' vocal signals, it does not capture the tone or content of this engagement. The sensitivity with which caregivers deliver language input may moderate the impact of AW and CT consistency on infants' risk for psychopathology. Clearly, compared to the complete absence of language input, the presence of any level of language input in an infants' environment is preferable; however, highly consistent input that is not attuned to the infant is unlikely to yield the best outcomes (see King, Humphreys, \& Gotlib, 2019, for an in-depth discussion of these issues). Future research may use the LENA or similar systems in tandem with coding of the content and tone of language input in relation to the infant's state in order to build a more nuanced understanding of infants' experiences and outcomes.

It is important to note that in addition to finding that infants who experience more consistent language input were at lower risk for emerging psychopathology, we found that infants' characteristics were associated with toddler symptoms. Replicating a large body of 
previous research indicating that infants with temperaments characterized by heightened negative affect in response to frustration and threat are more likely to develop psychopathology (Kostyrka-Allchorne et al., 2020), we found that infants who were reported by their mothers to be higher in negative emotionality had higher symptoms of psychopathology in toddlerhood. Further, we found that infants who vocalized more in their naturalistic environments had lower symptoms of psychopathology in toddlerhood. One potential explanation for this finding is that infants who vocalize more achieve earlier social and cognitive competencies that lead to a positive developmental cascade (Masten \& Cicchetti, 2010). For example, language is critical for communicating needs and forming close relationships and for understanding one's own and others' emotions and mental states-skills that help children regulate their attention, emotions, and behavior (Salmon, O'Kearney, Reese, \& Fortune, 2016). Indeed, poorer language skills in early childhood have been associated with higher symptoms of psychopathology across later childhood and adolescence (Bornstein, Chun-Shin, \& Suwalsky, 2013). Our study extends these findings to infancy and toddlerhood. Importantly, whereas infant characteristics are difficult to target directly in interventions designed to reduce risk for the development of psychopathology, features of infants' environments are mutable. Single features, such as aspects of language input, may have small effects on children's outcomes in the shorter-term; however, small effects can accumulate over time in ways that significantly alter susceptibility to life outcomes and costs to society (Funder \& Ozer, 2019). Although we do not suggest based on this single study that interventions should begin to target specifically increasing the consistency of language input, social policies that generally enhance caregivers' capacities to be available to and engage with their infants (e.g., paid parental leave) may have significant long-term effects on the wellbeing of the next generation.

Strengths of the current study include the longitudinal design and the multi-modal assessment of infants' environments. However, it will be important to replicate the current findings in different samples in different geographic areas. The sample in the current study was, 
on average, of higher socioeconomic status in terms of maternal education; further, mothers and fathers (or mothers' significant others) appeared to be the dominant source of adult speech in infants' environments. Although we observed wide variation in adult language input and maternal sensitivity even within this sample, these factors may affect the nature of caregiving and/or its impact on children. In addition, due to resource constraints, we used only maternal reports to assess child symptoms of psychopathology. Cross-informant integration may yield maximally accurate and useful information (Martel, Markon, \& Smith, 2017). In order to reduce participant burden and minimize demand characteristics and social desirability effects, we did not collect detailed diaries of the infants' activities concordant with the LENA recordings, precluding analysis of how infants' language input varies based on their schedules and routines. For example, we could not identify when infants were napping, and it is possible that variation in infants' nap schedules influenced measures of language input. Relatedly, we found that infants who engaged in their first CT earlier in the morning and in their last CT earlier in the evening had higher scores for both the quantity and consistency of language input, suggesting that infants' waking times and bedtimes may affect language input (see Supplementary Material). Investigating how infants' schedules and routines may influence their language environments is an important area for future research. Finally, our measures were based on a single day of recording of infants' language environments. Measures of language input obtained using the LENA have moderate test-retest reliability (Gilkerson et al., 2017), and repeated assessments of the language environment may yield more precise estimates of the association between language input and emerging psychopathology.

In conclusion, while our results should be replicated in future research, our findings underscore the importance of variation in the environment in typical family-based settings for emerging psychopathology in toddlerhood. In addition to infant and maternal characteristics, infants' experiences of the consistency of adult speech and vocal engagement with adults appear to be meaningfully related to their subsequent symptoms of psychopathology in 
toddlerhood. Although extant research examining infants' experiences of language input has focused primarily on cognitive outcomes, our findings highlight the importance of building a program of research that investigates variation in infants' naturalistic language environments in relation to the development of psychopathology. Ultimately, these findings may have important implications for the identification and measurement of features of the early environment that confer risk and resilience to psychopathology. 


\section{References}

Basten, M., Tiemeier, H., Althoff, R. R., van de Schoot, R., Jaddoe, V. W. V., Hofman, A., ... van der Ende, J. (2016). The stability of problem behavior across the preschool years: An empirical approach in the general population. Journal of Abnormal Child Psychology, 44(2), 393-404. https://doi.org/10.1007/s10802-015-9993-y

Belsky, J. (1980). Mother-infant interaction at home and in the laboratory: A comparative study. The Journal of Genetic Psychology. https://doi.org/10.1186/ar4524

Bornstein, M. H., Chun-Shin, H., \& Suwalsky, J. T. D. (2013). Language and internalizing and externalizing behavioral adjustment: Developmental pathways from childhood to adolescence. Development and Psychopathology, 25(3), 857-878. https://doi.org/10.1017/S0954579413000217

Bornstein, M. H., Putnick, D. L., Lansford, J. E., Pastorelli, C., Skinner, A. T., Sorbring, E., ... Oburu, P. (2015). Mother and father socially desirable responding in nine countries: Two kinds of agreement and relations to parenting self-reports. International Journal of Psychology, 50(3), 174-185. https://doi.org/10.1002/ijop.12084

Bosquet Enlow, M., Carter, A., Hails, K., King, L., \& Cabrera, I. (2014). Parent-Child Interaction Rating Scales - Infant Adaptation Manual.

Bowlby, J. (1988). A secure base: Parent-child attachment and healthy human development. The Journal of Nervous and Mental Disease. https://doi.org/10.1097/00005053$199001000-00017$

Bradley, R. H., \& Caldwell, B. M. (1984). The HOME Inventory and family demographics. Developmental Psychology, 20(2), 315-320. https://doi.org/10.1037/0012-1649.20.2.315

Briggs-Gowan, M. J., Carter, A. S., Bosson-Heenan, J., Guyer, A. E., \& Horwitz, S. M. (2006). Are infant-toddler social-emotional and behavioral problems transient? Journal of the American Academy of Child \& Adolescent Psychiatry, 45(7), 849-858. 
https://doi.org/10.1097/01.chi.0000220849.48650.59

Brito, N. H., Troller-renfree, S. V, Leon-santos, A., Isler, J. R., Fifer, W. P., \& Noble, K. G. (2020). Associations among the home language environment and neural activity during infancy. Developmental Cognitive Neuroscience, 43, 100780. https://doi.org/10.1016/j.dcn.2020.100780

Bronfenbrenner, U. (1977). Toward an experimental ecology of human development. American Psychologist, 32(7), 513-531. https://doi.org/10.1037/0003-066X.32.7.513

Calkins, S. D. (2011). Caregiving as coregulation: Psychobiological processes and child functioning. In Biosocial Foundations of Family Processes (pp. 49-59). https://doi.org/10.1007/978-1-4419-7361-0

Calkins, S. D., \& Hill, A. (2007). Caregiver influences on emerging emotion regulation: Biological and environmental transactions in early development. In Handbook of emotion regulation (pp. 229-248).

Camacho, M. C., King, L. S., Ojha, A., García, C. M., Sisk, L. S., Cichocki, A. C., ... Gotlib, I. H. (2020). Cerebral blood flow in 5- to 8-month-olds: Regional tissue maturity is associated with infant affect. Developmental Science. https://doi.org/10.1111/desc.12928

Carter, A. S., Briggs-Gowan, M. J., Jones, S. M., \& Little, T. D. (2003). The Infant-Toddler Social and Emotional Assessment (ITSEA): Factor structure, reliability, and validity. Journal of Abnormal Child Psychology, 31(5), 495-514. https://doi.org/10.1023/A:1025449031360

Chambers, J. M. (1992). Linear models. In J. M. Chambers \& T. J. Hastie (Eds.), Statistical Models in S. Wadsworth \& Brooks/Cole.

Cohen, J. (1977). Statistical power analysis for the behavioral sciences (rev. ed.). New York, NY: Academic Press.

Cohen, J. (1988). Statistical power analysis for the behavioral sciences (2nd ed.). Hillsdale, NJ: Erlbaum.

Cole, P. M., Martin, S. E., \& Dennis, T. A. (2018). Emotion regulation as a scientific construct: 
Methodological challenges and directions for child development research. Child Development, 75(2), 317-333.

Conradt, E., \& Ablow, J. (2010). Infant physiological response to the still-face paradigm:

Contributions of maternal sensitivity and infants' early regulatory behavior. Infant Behavior and Development, 33(3), 251-265. https://doi.org/10.1016/j.infbeh.2010.01.001

Cychosz, M., Romeo, R., Soderstrom, M., Scaff, C., Ganek, H., Cristia, A., ... Weisleder, A. (2020). Longform recordings of everyday life: Ethics for best practices. Behavior Research Methods. https://doi.org/10.3758/s13428-020-01365-9

Davidov, M., \& Grusec, J. E. (2006). Untangling the links of parental responsiveness to distress and warmth to child outcomes. Child Development, 77(1), 44-58.

Fernald, A., Marchman, V. A., \& Weisleder, A. (2013). SES differences in language processing skill and vocabulary are evident at 18 months. Developmental Science, 16(2), 234-248. https://doi.org/10.1111/desc.12019

Funder, D. C., \& Ozer, D. J. (2019). Evaluating effect size in psychological research: Sense and nonsense. Advances in Methods and Practices in Psychological Science, 2(2), 156-168. https://doi.org/10.1177/2515245919847202

Ganek, H., \& Eriks-Brophy, A. (2018). Language ENvironment analysis (LENA) system investigation of day long recordings in children: A literature review. Journal of Communication Disorders, 72, 77-85. https://doi.org/10.1016/j.jcomdis.2017.12.005

Gilkerson, J., \& Richards, J. (2008). The LENA natural language study. Boulder, CO: The LENA Foundation. Retrieved from http://www.lenafoundation.org/Research/TechnicalReports.aspx

Gilkerson, J., Richards, J. A., Warren, S. F., Montgomery, J. K., Greenwood, C. R., Kimbrough Oller, D., ... Paul, T. D. (2017). Mapping the early language environment using all-day recordings and automated analysis. American Journal of Speech-Language Pathology, 26(2), 248. https://doi.org/10.1044/2016_AJSLP-15-0169 
Gilkerson, J., Richards, J. A., Warren, S. F., Oller, D. K., Russo, R., \& Vohr, B. (2018).

Language experience in the second year of life and language outcomes in late childhood. Pediatrics, 142(4). https://doi.org/10.1542/peds.2017-4276

Grant, K. A., McMahon, C., Reilly, N., \& Austin, M. P. (2010). Maternal sensitivity moderates the impact of prenatal anxiety disorder on infant responses to the Still-Face Procedure. Infant Behavior and Development, 33(4), 453-462. https://doi.org/10.1016/j.infbeh.2010.05.001

Gunnar, M. R., Van Dulmena, M. H. ., \& The International Adoption Project Team. (2007). Behavior problems in postinstitutionalized internationally adopted children. Development and Psychopathology, 19(01), 129-148. https://doi.org/10.1017/S0954579407070071

Haley, D. W., \& Stansbury, K. (2003). Infant stress and parent responsiveness: Regulation of physiology and behavior during still-face and reunion. Child Development, 74(5), 15341546.

Harris, P. A., Taylor, R., Minor, B. L., Elliot, V., Fernandez, M., O’Neal, L., ... REDCap Consortium. (2019). The REDCap consortium: Building an international community of software partners. Journal of Biomedical Informatics, 95. https://doi.org/https://doi.org/10.1016/j.jbi.2019.103208

Harris, P. A., Taylor, R., Thielke, R., Payne, J., Gonzales., N., \& Conde, J. G. (2009). Research electronic data capture (REDCap) - A metadata-driven methodology and workflow process for providing translational research informatics suppor. Journal of Biomedical Informatics, 42(2), 377-381. https://doi.org/https://doi.org/10.1016/j.jbi.2008.08.010

Hawk, B., \& McCall, R. B. (2010). CBCL behavior problems of post-institutionalized international adoptees. Clinical Child and Family Psychology Review, 13(2), 199-211. https://doi.org/10.1007/s10567-010-0068-x

Humphreys, K. L., Gleason, M. M., Drury, S. S., Miron, D., Nelson, C. A., Fox, N. A., \& Zeanah, C. H. (2015). Effects of institutional rearing and foster care on psychopathology at age 12 years in Romania: Follow-up of an open, randomised controlled trial. The Lancet 
Psychiatry, 2(7), 625-634. https://doi.org/10.1016/S2215-0366(15)00095-4

Humphreys, K. L., King, L. S., Choi, P., \& Gotlib, I. H. (2018). Maternal depressive symptoms, self-focus, and caregiving behavior. Journal of Affective Disorders, 238, 465-471. https://doi.org/10.1016/j.jad.2017.07.051

Humphreys, K. L., King, L. S., \& Gotlib, I. H. (2018). Neglect. In C. H. Zeanah (Ed.), Handbook of Infant Mental Health.

Humphreys, K. L., \& Zeanah, C. H. (2015). Deviations from the expectable environment in early childhood and emerging psychopathology. Neuropsychopharmacology, 40(1), 154-170. https://doi.org/10.1038/npp.2014.165

Jerome, F., Hastie, T., Simon, N., \& Tibshirani, R. (2010). Regularization paths for generalized linear models via coordinate descent. Journal of Statistical Software, 33(1), 1-22. Retrieved from http://www.jstatsoft.org/v33/i01/

Johnson, S. C., Dweck, C. S., \& Chen, F. S. (2016). Evidence for infants' internal working models of attachment. Psychological Science, 18(6), 501-502.

Kass, R. E., \& Raftery, A. E. (1995). Bayes factors. Journal of the American Statistical Association, 90(430), 319-323. https://doi.org/10.1108/10775730610619007

King, L. S., Camacho, M. C., Montez, D. F., Humphreys, K. L., \& Gotlib, I. H. (2020). Naturalistic language input is associated with resting-state functional connectivity in infancy.

King, L. S., Humphreys, K. L., \& Gotlib, I. H. (2019). The neglect-enrichment continuum: Characterizing variation in early caregiving environments. Developmental Review, 51, 109122. https://doi.org/10.1016/j.dr.2019.01.001

Kok, R., Linting, M., Bakermans-Kranenburg, M. J., van ljzendoorn, M. H., Jaddoe, V. W. V., Hofman, A., ... Tiemeier, H. (2013). Maternal sensitivity and internalizing problems: Evidence from two longitudinal studies in early childhood. Child Psychiatry and Human Development, 44(6), 751-765. https://doi.org/10.1007/s10578-013-0369-7

Kostyrka-Allchorne, K., Wass, S. V., \& Sonuga-Barke, E. J. S. (2020). Research Review: Do 
parent ratings of infant negative emotionality and self-regulation predict psychopathology in childhood and adolescence? A systematic review and meta-analysis of prospective longitudinal studies. Journal of Child Psychology and Psychiatry, 61(4), 401-416. https://doi.org/10.1111/jcpp.13144

Leerkes, E. M. (2011). Maternal sensitivity during distressing tasks: A unique predictor of attachment security. Infant Behavior and Development, 34(3), 443-446. https://doi.org/10.1016/j.infbeh.2011.04.006

Leerkes, E. M., Blankson, A., \& O’Brien, M. (2009). Differential effects of maternal sensitivity to infant distress and non-distress on social-emotional functioning. Child Development, 80(3), 762-775. https://doi.org/10.1111/j.1467-8624.2009.01296

LeMoult, J., Humphreys, K. L., Tracy, A., Hoffmeister, J. A., Ip, E., \& Gotlib, I. H. (2020). Metaanalysis: Exposure to early life stress and risk for depression in childhood and adolescence. Journal of the American Academy of Child and Adolescent Psychiatry, 59(7), 842-855. https://doi.org/10.1016/j.jaac.2019.10.011

Martel, M. M., Markon, K., \& Smith, G. T. (2017). Research Review: Multi-informant integration in child and adolescent psychopathology diagnosis. Journal of Child Psychology and Psychiatry and Allied Disciplines, 58(2), 116-128. https://doi.org/10.1111/jcpp.12611

Masten, A. S., \& Cicchetti, D. (2010). Developmental cascades. Development and Psychopathology, 22(3), 491-495. https://doi.org/10.1017/S0954579410000222

McGoron, L., Gleason, M. M., Smyke, A. T., Drury, S. S., Nelson, C. A., Gregas, M. C., ... Zeanah, C. H. (2012). Recovering from early deprivation: Attachment mediates effects of caregiving on psychopathology. Journal of the American Academy of Child and Adolescent Psychiatry, 51(7), 683-693. https://doi.org/10.1016/j.jaac.2012.05.004

McLaughlin, K. A., Sheridan, M. A., \& Nelson, C. A. (2017). Neglect as a violation of speciesexpectant experience: Neurodevelopmental consequences. Biological Psychiatry, 82(7), 462-471. https://doi.org/10.1016/j.biopsych.2017.02.1096 
Mehl, M. R. (2017). The Electronically Activated Recorder (EAR): A Method for the Naturalistic Observation of Daily Social Behavior. Current Directions in Psychological Science, 26(2), 184-190. https://doi.org/10.1177/0963721416680611

Merz, E. C., Maskus, E. A., Melvin, S. A., He, X., \& Noble, K. G. (2019). Socioeconomic disparities in language input are associated with children's language-related brain structure and reading skills. Child Development, 1-15. https://doi.org/10.1111/cdev.13239

Mesman, J., van IJzendoorn, M. H., \& Bakermans-Kranenburg, M. J. (2009). The many faces of the Still-Face Paradigm: A review and meta-analysis. Developmental Review, 29(2), 120 162. https://doi.org/10.1016/j.dr.2009.02.001

Morey, R. D. (2019). Computation of Bayes factors for common designs [R package BayesFactor version 0.9.12-4.2]. CRAN Repository. Retrieved from https://cran.rproject.org/web/packages/BayesFactor/index.html

Peverill, M., Dirks, M. A., Narvaja, T., Herts, K. L., Comer, J. S., \& McLaughlin, K. A. (2021). Socioeconomic status and child psychopathology in the United States: A meta-analysis of population-based studies. Clinical Psychology Review, 83(May 2020), 101933. https://doi.org/10.1016/j.cpr.2020.101933

Putnam, S. P., Helbig, A. L., Gartstein, M. A., Rothbart, M. K., \& Leerkes, E. (2014). Development and assessment of short and very short forms of the Infant Behavior Questionnaire-Revised. Journal of Personality Assessment, 96(4), 445-458. https://doi.org/10.1080/00223891.2013.841171

Quintana, D. S., \& Williams, D. R. (2018). Bayesian alternatives for common null-hypothesis significance tests in psychiatry: A non-technical guide using JASP. BMC Psychiatry, 18178. https://doi.org/10.1186/s12888-018-1761-4

R Core Team. (2020). R: A language and environment for statistical computing. Vienna, Austria: R Foundation for Statistical Computing. Retrieved from https://www.r-project.org/ Radloff, L. S. (1977). The CES-D scale: A self-report depression scale for research in the 
general population. Journal of Applied Psychological Measurement, 1, 385-401.

Richards, J. A., Gilkerson, J., Xu, D., \& Topping, K. (2017). How much do parents think they talk to their child? Journal of Early Intervention, 39(3), 163-179. https://doi.org/10.1177/1053815117714567

Romeo, R. R., Leonard, J. A., Robinson, S. T., West, M. R., Mackey, A. P., Rowe, M. L., \& Gabrieli, J. D. E. (2018). Beyond the 30-million-word gap: Children's conversational exposure is associated with language-related brain function. Psychological Science, 29(5), 700-710. https://doi.org/10.1177/0956797617742725

Romeo, R. R., Segaran, J., Leonard, J. A., Robinson, S. T., West, M. R., Mackey, A. P., ... Gabrieli, J. D. E. (2018). Language exposure relates to structural neural connectivity in childhood. The Journal of Neuroscience, 38(36), 0484-18. https://doi.org/10.1523/JNEUROSCI.0484-18.2018

Salmon, K., O'Kearney, R., Reese, E., \& Fortune, C. A. (2016). The role of language skill in child psychopathology: Implications for intervention in the early years. Clinical Child and Family Psychology Review, 19(4), 352-367. https://doi.org/10.1007/s10567-016-0214-1

Salo, V. C., Pannuto, P., Hedgecock, W., Biri, A., Russo, D. A., Piersiak, H., \& Humphreys, K. L. (2020). Measuring naturalistic proximity as a window into caregiver-child interaction patterns. https://doi.org/10.31234/osf.io/h2fy6

Sherman, L. J., Rice, K., \& Cassidy, J. (2015). Infant capacities related to building internal working models of attachment figures: A theoretical and empirical review. Developmental Review (Vol. 37). Elsevier Inc. https://doi.org/10.1016/j.dr.2015.06.001

Sosinsky, L. S., Marakovitz, S., \& Carter, A. S. (2004). Parent-Child Interaction Rating Scales (PCIRS). Unpublished Manual. University of Massachusetts Boston.

Stoltenborgh, M., Bakermans-Kranenburg, M. J., \& Van ljzendoorn, M. H. (2013). The neglect of child neglect: A meta-analytic review of the prevalence of neglect. Social Psychiatry and Psychiatric Epidemiology, 48(3), 345-355. https://doi.org/10.1007/s00127-012-0549-y 
Tamis-LeMonda, C. S., Kuchirko, Y., Luo, R., Escobar, K., \& Bornstein, M. H. (2017). Power in methods: Language to infants in structured and naturalistic contexts. Developmental Science, 20(6). https://doi.org/10.1111/desc.12456

Tronick, E., Als, H., Adamson, L., Wise, S., \& Brazelton, T. B. (1978). The infant's response to entrapment between contradictory messages in face-to-face interaction. Journal of the American Academy of Child Psychiatry, 17(1), 1-13. JOUR.

van Aar, J., Leijten, P., Orobio de Castro, B., \& Overbeek, G. (2017). Sustained, fade-out or sleeper effects? A systematic review and meta-analysis of parenting interventions for disruptive child behavior. Clinical Psychology Review, 51, 153-163. https://doi.org/10.1016/j.cpr.2016.11.006

Vasileva, M., Graf, R. K., Reinelt, T., Petermann, U., \& Petermann, F. (2020). Research review: A meta-analysis of the international prevalence and comorbidity of mental disorders in children between 1 and 7 years. Journal of Child Psychology and Psychiatry, 0-5. https://doi.org/10.1111/jcpp.13261

Weisleder, A., \& Fernald, A. (2013). Talking to children matters: Early language experience strengthens processing and builds vocabulary. Psychological Science, 24(11), 2143-2152. https://doi.org/10.1177/0956797613488145

Wright, N., Hill, J., Sharp, H., \& Pickles, A. (2018). Maternal sensitivity to distress, attachment and the development of callous-unemotional traits in young children. Journal of Child Psychology and Psychiatry, 59(7), 790-800. https://doi.org/10.1111/JCPP.12867

Xu, D., Yapanel, U., \& Gray, S. (2009). Reliability of the LENA language environment analysis system in young children's natural home environment. Retrieved from https://www.lena.org/wp-content/uploads/2016/07/LTR-05-2_Reliability.pdf

Yap, M. B. H., Morgan, A. J., Cairns, K., Jorm, A. F., Hetrick, S. E., \& Merry, S. (2016). Parents in prevention: A meta-analysis of randomized controlled trials of parenting interventions to prevent internalizing problems in children from birth to age 18. Clinical Psychology Review, 
50, 138-158. https://doi.org/10.1016/j.cpr.2016.10.003

Zeanah, C. H., Smyke, A. T., \& Settles, L. (2006). Children in orphanages. In K. McCartney \& D. Phillips (Eds.), Blackwell Handbook of Early Childhood Development (pp. 224-254). Malden, MA: Blackwell Publishing.

Zeanah, C. H., \& Zeanah, P. D. (2018). Infant mental health: The clinical science of early Experience. In C. H. Zeanah (Ed.), Handbook of Infant Mental Health (4th Editio). New York: Guilford Press.

Zero to Three. (2016). DC:0-5: Diagnostic Classification of Mental Health and Developental Disorders of Infancy and Early Childhood. Washington, DC. 
Table 1. Descriptive statistics. $N=100 .{ }^{1}$ Annual household income ranged from $\$ 30,001-60,000$ to $>\$ 150,000$, with $22 \%$ classified as "low-income" based on an income-to-needs ratio < 1 calculated with respect to the Department of Housing and Urban Development's low-income limits for the county in which data were collected. ${ }^{2}$ Total recording duration is the length in hours of the raw LENA recording. The start and ends times of the analyzed recording duration corresponded to the first and last conversational turn in the recording. ${ }^{3}$ At the toddler assessment, number of children with symptoms of depression/withdrawal, general anxiety, activity/impulsivity, aggression/defiance, or negative emotionality above age and gendernormed cutoffs for clinical concern.

\begin{tabular}{|c|c|c|}
\hline Measure & $\underset{N}{\operatorname{Mean}(S D)}$ or & Range \\
\hline $\begin{array}{l}\text { Maternal age (years) } \\
\text { Child age (months) }\end{array}$ & $33.74(4.67)$ & $19.51-45.63$ \\
\hline Infant assessment & $6.73(0.75)$ & $5.19-8.74$ \\
\hline Toddler assessment & $18.29(0.52)$ & $17.52-21.15$ \\
\hline Income-to-needs ratio ${ }^{1}$ & $1.48(0.52)$ & $0.13-2.21$ \\
\hline Infant negative emotionality & $3.14(0.76)$ & $1.63-4.98$ \\
\hline $\begin{array}{l}\text { Infant vocalization maximum } \\
\text { Maternal depressive symptoms }\end{array}$ & $61.17(20.84)$ & $30-132$ \\
\hline Infant assessment & $9.05(8.91)$ & $0-43$ \\
\hline Toddler assessment & $8.04(7.31)$ & $0-33$ \\
\hline Naturalistic language input & & \\
\hline Total recording duration (hours) ${ }^{2}$ & $15.19(1.44)$ & $8.49-16.00$ \\
\hline Analyzed recording duration (hours) ${ }^{2}$ & $12.09(1.72)$ & $7.67-15.58$ \\
\hline Analyzed start time (HH:MM) & $7: 40$ AM $(1: 19)$ & 5:00 AM-1:34 PM \\
\hline Analyzed end time (HH:MM) & 8:19 PM (1:29) & 5:10 PM-11:55 PM \\
\hline Adult word quantity & $3508.51(1451.11)$ & $593-7788$ \\
\hline Adult word consistency & $0.74(0.11)$ & $0.46-0.97$ \\
\hline Conversational turn quantity & $74.13(30.73)$ & $14-174$ \\
\hline Conversational turn consistency & $0.55(0.12)$ & $0.20-0.81$ \\
\hline $\begin{array}{l}\text { Infant vocalizations } \\
\text { L }\end{array}$ & $257.59(107.24)$ & $83-617$ \\
\hline $\begin{array}{l}\text { Laboratory caregiving behavior } \\
\text { Maternal sensitivity to distress }\end{array}$ & $4.12(1.06)$ & \\
\hline Maternal sensitivity during play & $3.81(0.85)$ & $\begin{array}{l}1.69-6.13 \\
1.75-6.00\end{array}$ \\
\hline $\begin{array}{l}\text { Toddler symptoms of } \\
\text { psychopathology }\end{array}$ & $1.06(0.50)$ & $0.19-2.38$ \\
\hline $\begin{array}{l}\text { Toddler \% clinical concern } \\
\text { Infant male sex }\end{array}$ & $\begin{array}{l}20 \\
55\end{array}$ & \\
\hline Maternal Hispanic/Latinx ethnicity & 15 & \\
\hline Maternal race & & \\
\hline White & 65 & \\
\hline Asian or Asian American & 22 & \\
\hline Black or African American & 3 & \\
\hline Native Hawaiian or Pacific Islander & 2 & \\
\hline Other race & 8 & \\
\hline Maternal education & & \\
\hline$<4$-year college & 13 & \\
\hline Bachelor's degree & 36 & \\
\hline Graduate degree & 51 & \\
\hline
\end{tabular}


Table 2. Linear regression results predicting symptoms of psychopathology in toddlerhood. Degrees of freedom $=92$. Each measure of language input was tested in a separate regression model including the covariates of maternal depressive symptoms at the toddler assessment and infant negative emotionality. Bayes Factors indicate the strength of evidence in favor of the alternative hypothesis that the measure of language input is associated with symptoms of psychopathology above and beyond the covariates. Bayes Factors ranging from 1-3 are considered "anecdotal" or "barely worth mention" evidence; Bayes factors ranging from 3-10 are considered "moderate" or "positive" evidence (Kass \& Raftery, 1995; Quintana \& Williams, 2018).

\begin{tabular}{cccccc}
\hline $\begin{array}{c}\text { Dimension of language } \\
\text { environment }\end{array}$ & $\boldsymbol{\beta}[95 \% \mathrm{Cl}]$ & $\boldsymbol{t}$ & $\boldsymbol{p}$ & $\boldsymbol{R}^{2}$ & Bayes Factor \\
\hline $\begin{array}{c}\text { Consistency of input } \\
\text { Adult words }\end{array}$ & $\begin{array}{c}-0.22 \\
{[-0.41,-0.03]} \\
-0.25\end{array}$ & -2.32 & .023 & .05 & 3.00 \\
Conversational turns & -2.63 & .010 & .06 & 5.79 \\
$\begin{array}{c}{[-0.43,-0.06]} \\
\text { Quantity of input } \\
\text { Adult words }\end{array}$ & $\begin{array}{c}-0.06 \\
{[-0.25,0.13]} \\
-0.22\end{array}$ & -0.62 & .537 & $<.01$ & 0.35 \\
Conversational turns & $\begin{array}{c}-0.28 \\
{[-0.41,-0.03]} \\
-0.24\end{array}$ & -2.28 & .025 & .03 & 2.80 \\
Infant vocalizations & -2.54 & .013 & .05 & 4.72 \\
\hline
\end{tabular}


Table 3. Elastic net regression results predicting symptoms of psychopathology in toddlerhood. $\mathrm{N}=96$. AW = adult words. $\mathrm{CT}=$ conversational turns. Maternal depressive symptoms were measured at the toddler assessment. The coefficients of correlated predictors are shrunk towards each other; thus, although the coefficients are standardized, lower coefficients do not reflect smaller zero-order effect sizes. $R^{2}$ Total $=.24$.

\begin{tabular}{lcc}
\hline \multicolumn{1}{c}{ Variable } & Elastic net estimate & $\begin{array}{c}\text { Zero-order effect size } \\
\text { (Pearson's } \boldsymbol{r} \text { ) }\end{array}$ \\
\hline (Intercept) & 0.00 & \\
CT consistency & -0.02 & -.24 \\
AW consistency & -0.15 & -.22 \\
CT quantity & -0.06 & -.13 \\
Infant vocalizations & -0.14 & -.20 \\
Maternal depressive & 0.19 & .20 \\
symptoms & & .34 \\
Infant negative affectivity & 0.30 & \\
\hline
\end{tabular}


Figure 1. Correlations among naturalistic measures of language input and laboratorybased observations of maternal sensitivity in infancy. $A W=$ adult words. $C T=$ conversational turns. Coefficients are calculated using pairwise deletion of missing data $\left(N_{\text {Language Input Variables }}=100, N_{\text {Sensitivity During Play }}=96, N_{\text {Sensitivity to Distress }}=94\right)$.

Sensitivity to distress

Sensitivity during play

Infant vocalizations

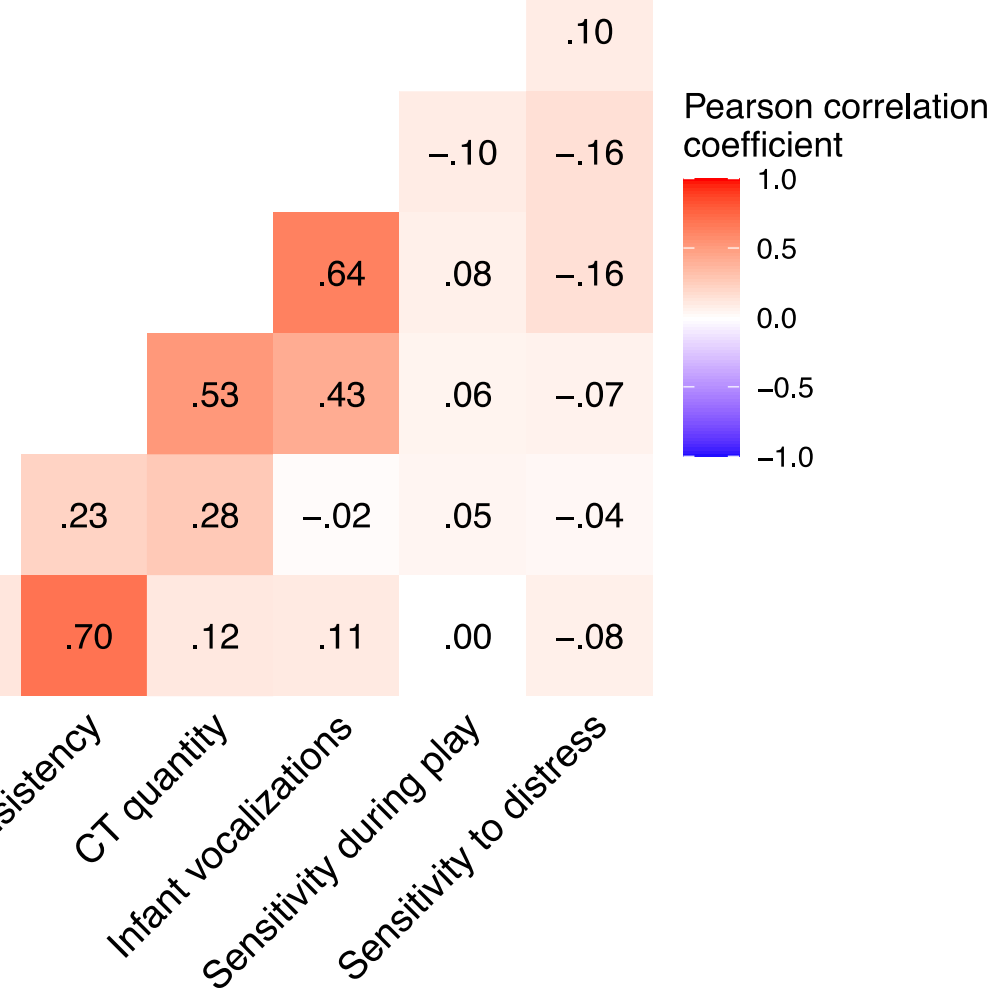


Figure 2. Distinguishing the quantity versus the consistency of language input in the daily language environment. The distribution of conversational turns in each 5-minute epoch of the day for two infants: one exposed to less numerous but more consistent conversational turns (red); and one exposed to more numerous but less consistent conversational turns (blue).

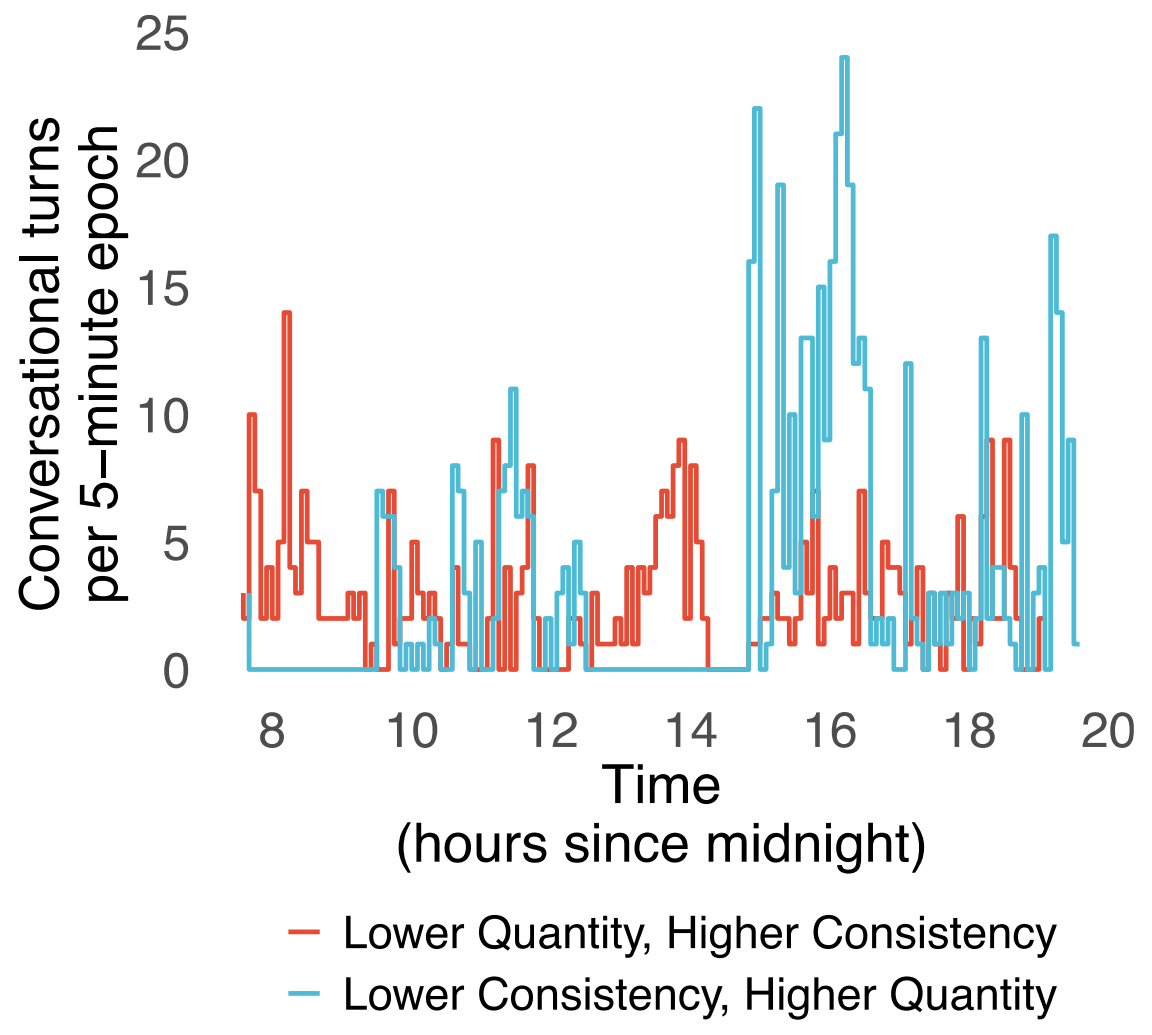


Figure 3. The consistency of adult words and conversational turns in infancy is negatively associated with symptoms of psychopathology in toddlerhood. Toddler symptoms of psychopathology are residuals after covarying for infant negative emotionality and maternal depressive symptoms at the toddler assessment. Consistency was analyzed as a proportion and shown here converted to a percentage. A score of $50 \%$ indicates that the infant experienced $\geq 1$ adult word or conversational turn for $50 \%$ of the five-minute epochs in the recording day.

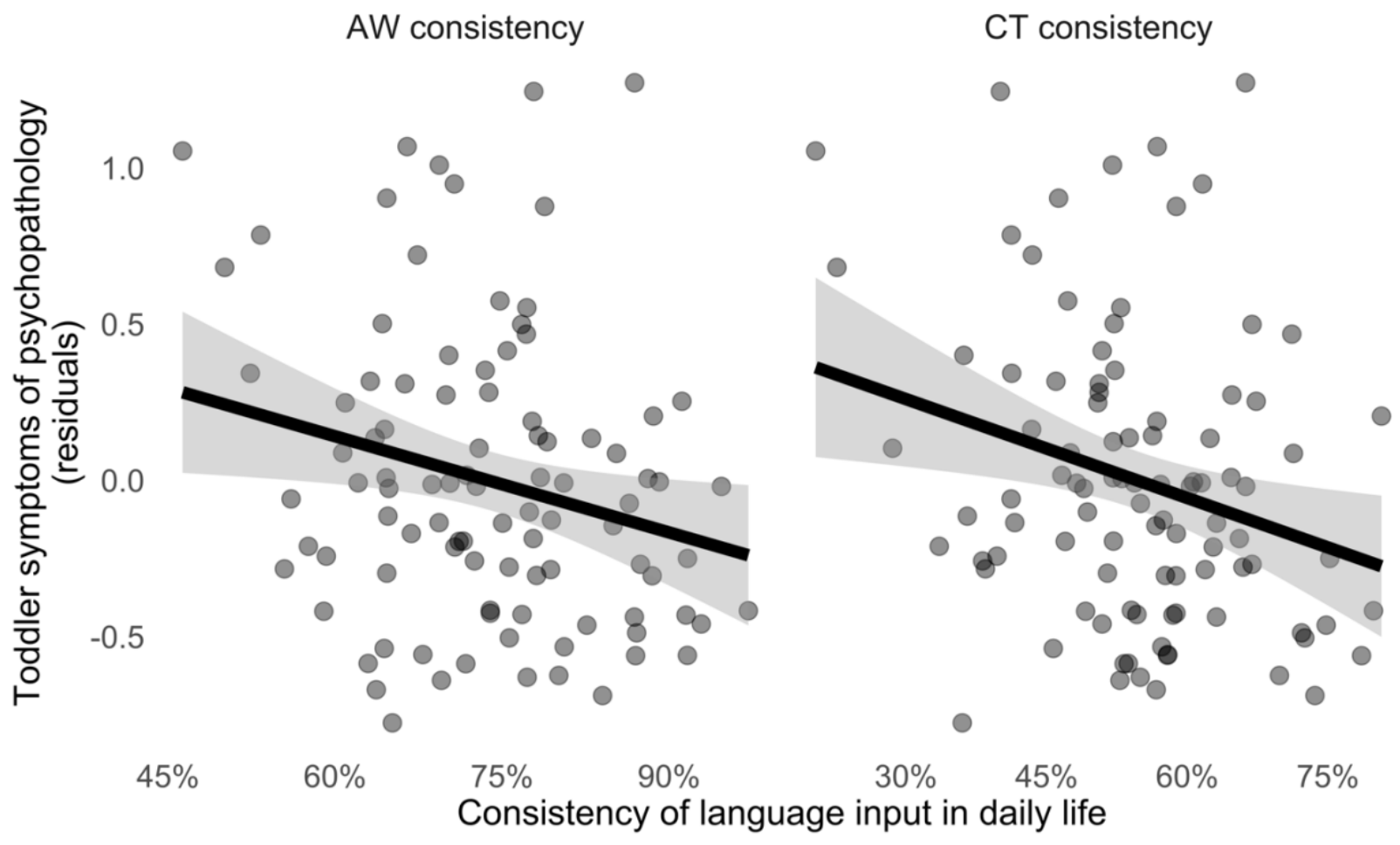

